\title{
VARIACIÓN EST ACIONAL DE GRANDES MEDUSAS (SCYPHOZOA) EN UN SISTEMA DE LAGUNAS COSTERAS DEL SUR DEL GOLFO DE MEXICO (1977/1978)
}

SAMUEL GOMEZ-AGUIRRE

Instituto de Biología, Universidad Autónoma de México México 20, D. F. Mẹ́xico

\section{SYNOPSIS}

Two species of large jelly-fish,' Aurelia aurita $L$. and Stomolophus meleagris Ag. have been registered within a yearly cycle at the system of coastal lagoons "Carmen-Machoria-Redonda", South of the Gulf of Mexico. A. aurita showed two well defined periods: autumn/winter with predominant young specimens $(25$ to $100 \mathrm{~mm}$ ) and spring/summer with a wide adult distribution $(200$ to $400 \mathrm{~mm})$, beeing the juveniles restricted to the internal regions of the system. S. meleagris gave evidence of adult populations all year round, the greatest population density occurred during the summer/autumn period with maximum sizes of 200 to $300 \mathrm{~mm}$ and during the winter/spring period, juveniles from 10 to $30 \mathrm{~mm}$ in December and 50 to $100 \mathrm{~mm}$ in A pril, giving a distribution pattern very similar to A, surita.

\section{Introducción}

Durante más de una década de estudios continuados del plancton de diversas lagunas costeras del Sur y Suroeste del Golfo de México, hemos confrontado la importancia de las scyphomedusas como habitantes temporales o circunstanciales de estos lugares. Sin embargo, por diversas razones, su estudio se ha venido aplazando en principio porque los programas a desarrollar las han excluido -no por ser menos importantes -sino por darles prioridad a otros aspectos de la planctologia: ejem. fitoplancton, zooplancton, productividad primaria, biomasa etc, No obstante, como ya se ha expuesto en ocasión anterior (Gómez-Aguirre, 1975), se han hecho algunas contribuciones a la biología y ecología de esta clase de los celenterados, pretendiendo incluso abordar algunos aspectos de la genética y los fenómenos de regeneración.

La presencia de scyphomedusas en lagunas costeras del Golfo de México se habia registrado como un fenómene temporal breve o más o menos amplio, regulado fundamentalmente por el grado de influencia marina y por la estabilidad ambiental de las mismas.

En nuestros reconocimientos de prospección hidrobiológica en 1976, para iniciar el estudio del ciclo anual del plancton y la productividad del sistema de lagunas costeras que se localiza en el flánco S. SE de los $18^{\circ} 20^{\prime} \mathrm{N}$ y los $93^{\circ} 50^{\prime} \mathrm{W}$ y que está formado por cuatro lagunas intercomunicadas con dos cuerpos principales: El Carmen y La Machona. cada cual con una laquna menor o remanente de su antigua configuración y que son La Palma en el extremo Sur de la Laguna del Carmen y La Redonda situada al Noreste de la Laguna La Machona (Fig. 1), nos percatamos de la presencia de una importante población de Stomolophus meleagris Ag. y una menor existencia de Aurelia aurita L., hecho que consideramos normal según nuestras experiencias previas en otras áreas similares. (Gómez-Aguirre, en prensa).

Al iniciar nuestros reconocimientos periódicos y muestros biológicos de este sistema de lagunas costeras, pudimos estimar a groso modo, los enormes volúmenes representados por la población de $S$. meleagris que en forma muy superficial se calcularon en aproximadamente $30 \times 10^{6}$. Este fenómeno nos atrajo más cuando nos enteramos de que tal abundancia de medusas grandes era de reciente aparición y su origen se atribuía a la abertura de una comunicación artificial en la Barra de Alacranes en diciembre de 1975: (Reséndez-Medina, 1980). Efectivamente, anteriores estudios biológicos de la región ( * ) no hacen mención alguna a la existencia de medusas grandes.

En la necesidad de conocer el papel ecológico así como el ciclo biológico ae $\mathrm{S}$. meieagrys y $A$, aurita en este sistema lagunar, nos propusmos mantener muestreos, uoservaciones y estumaciones cuantitativas periódicas de estas poblaciones, paralelas con las de hidrología, plancton y peces (Reséndez-Medina, op. cit.), cada dos meses durante un cicio anual comprendido entre Agosto de 1977 y Julio de 1978.

Nuestro interés por presentar esta pequeña contribución deriva del hecho de que es la primera vez que observamos este fenómeno tan marcado en lagunas costeras en que se trata de beneficiar y controlar las características del medio ambiente para favorecer los cultivos de ostras e incrementar la producción de crustáceos y peces.

(*) Tesis de licenciatura - Facultad de Ciencias, Universidad Nacional Autónoma de México (varios autores) - 1972/1974.
Area de Estudio

E1 sistema de lagunas costeras 'Carmen-Machona-Redonda', se localiza en el extremo sur del Golfo de México y propiamente corresponde al proceso costero Norte del Istmo de Tehuantepec (la región más estrecha de México), que en su costa sur presenta dos sistemas gemelos de dimensiones algo mayores y de configuración muy semejante, de gran atractivo para investigaciones sobre ta geologia y biología.

Una excelente referencia fisiográfica y toponímica de estos cuerpos de agua la expone Reséndez-Medina (op. cit.), en su trabajo sobre la hidrología de estas mismas áreaś en el mismo ciclo anual, señalando que se trata de áreas salobres con gran influencia marina y de muy escasa variación hidrológica por lo menos durante el plazo de nuestras investigaciones.

\section{Material y Metodo}

Por experiencias previas en los reconocimientos de Stomolophus meleagris y de Aurelia aurita se estimó innecesario realizar colectas específicas y sólo eventualmente se extrajeron especímenes por medio de redes de cuchara para reconocimiento general, se conservaron o bien se transportaban vivos a acuarios para etectuar observaciones de comportamiento, examinar posibles traumas y procesos de regeneración, temas de los cuales se tienen algunos planteamientos para estudios posteriores.

El agua transparente que varía entre 0.8 y $3.0 \mathrm{~m}$ de Disco de Secchi en aproximadamente el $90 \%$ del área del sistema Carmen-Machona-Redonda, permitió el reconocimiento de $S$. $m e$. leagris y A. aurita a cierta distancia y a una profundidad entre 0 y $0.5 \mathrm{~m}$ de profundidad. En tales circunstancias se hicieron cuantiticaciones en una faja de $10 \times 100 \mathrm{~m}$ en línea y su resultado posteriormente se empleó para calcular el número de individuos por Hectárea. Estos resultados fueron ponderados y transportados a tres cartas de distribución y abundancia de $S$. meleagris y $A$. aurita, sintetizándose en dos grandes épocas: verano/otoño e invierno/primavera para $S$. meleagris, debido a que su comportamiento fue muy semejante en los intervalos de dos meses; para $A$. aurita sólo en una época - primavera/verano - en que fueron mas constantes los adultos.

\section{Resultados y Discusion}

Aunque la información obtenida durante el período de nuestros estudios (agosto de 1977 a julio de 1978), es bastante amplia, esta oportunidad sólo nos permite referirnos al aspecto más grueso y sobresaliente de la variación estacional de Stomo lophus meleagris y Aurelia aurita, dejando para posterior ocasión la exposición y discusión global del papel que juegan las scyphomedusas en sistemas de lagunas costeras del Sur del Golfo de México y regiones adyacentes del Mar Caribe.

\section{Stomolophus meleagris Ag.}

Los resultados sobre esta medusa nos permiten establecer dos épocas diferentes en el año, una - verano/otoño - con predominancia de adultos maduros y otra correspondiente a invierno/primavera, con una altísima población de formas juveniles. En la primera de estas dos épocas, nuestro método de observación nos permitió distinguir cuatro categorias de la abundancia, como se ilustra en la Figura 1, en la cual se incluyen las observaciones de los meses de agosto y octubre de 1977 y julio de 1978. En es- 


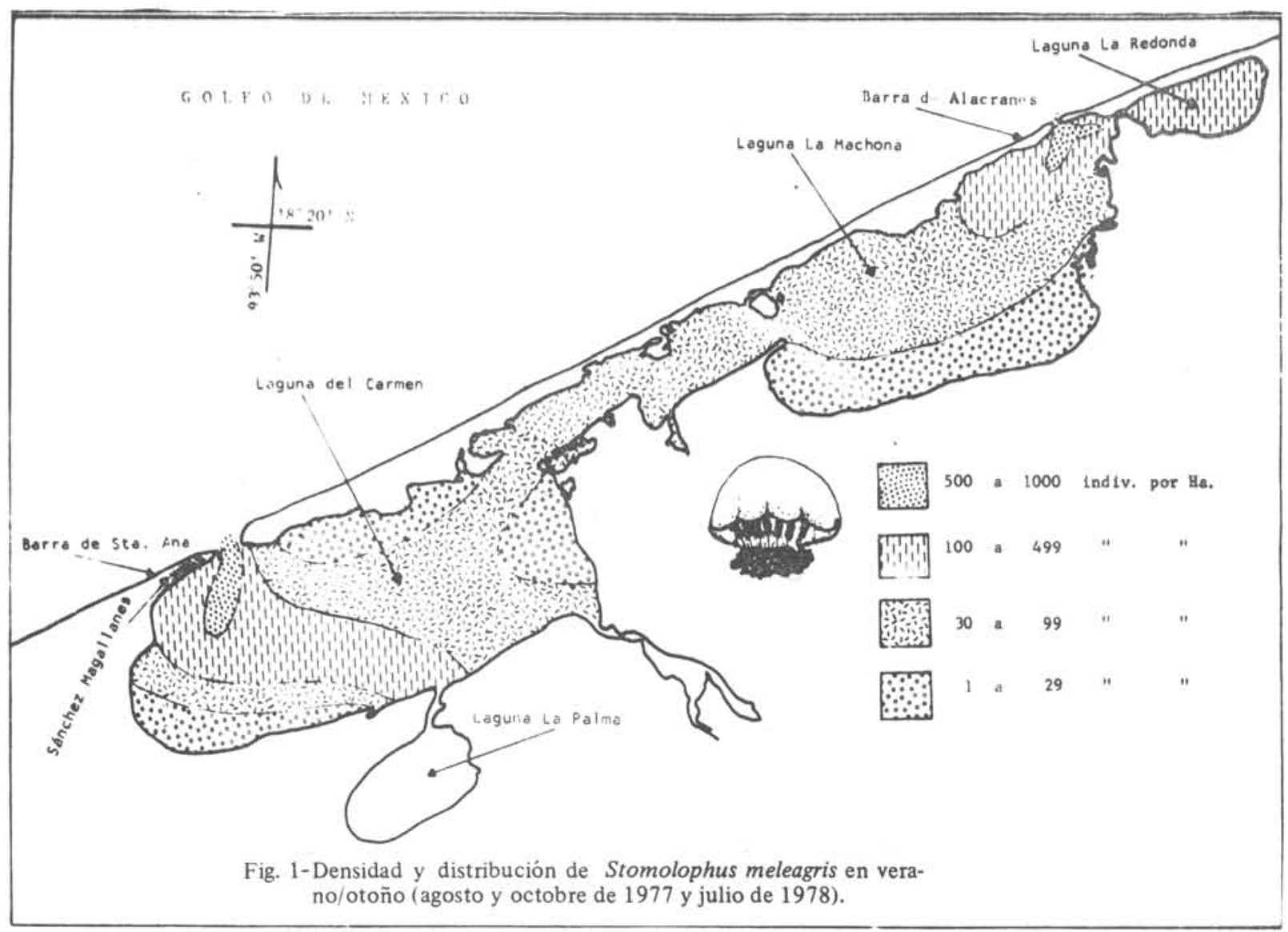

te período del ciclo anual se calcularon densidades de 500 a 1000 medusas por Hectárea en el radio de las 3 a las 5 millas próximas a las bocas del Carmen y de Alacranes, respectivamente, a partir de donde se dispersan a todo el sistema, llegando a observarse algunas concentraciones de estas poblaciones en áreas confinadas como en la Laguna Redonda y La Palma así como en algunos sitios del
Canal que comunica a las lagunas Del Carmen y La Machona. La mayor superficie del sistema mostró densidades estimadas entre 30 a 99 indiv. por $\mathrm{Ha}$., en las áreas aledañas a los canales y entre 100 a 499 indiv. por Ha., en las regiones de influencia de las bocas.

En el período invierno/primavera (Fig. 2), se apreció un notorio descenso de la población, que atribuímos a dos causas funda-

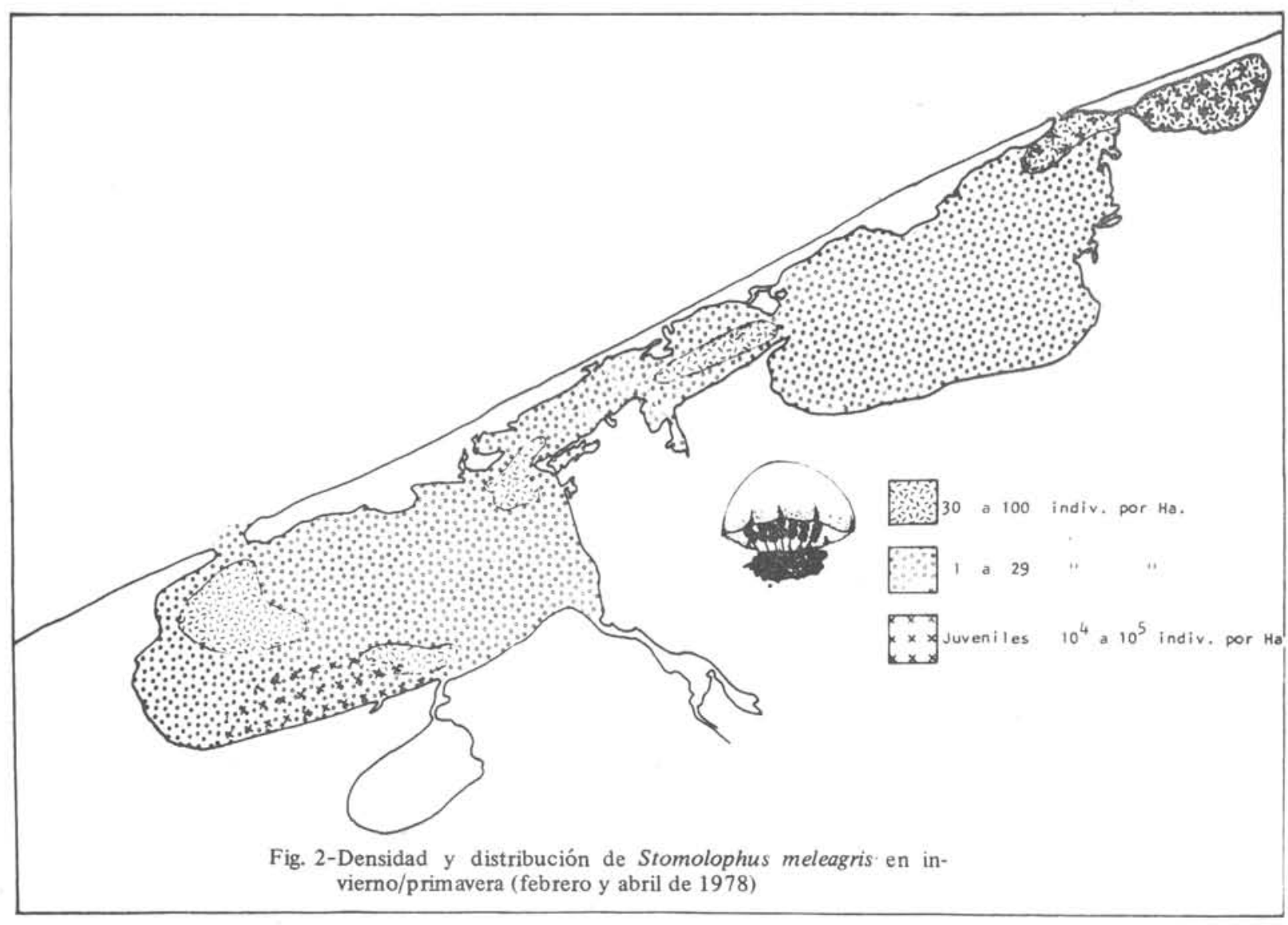




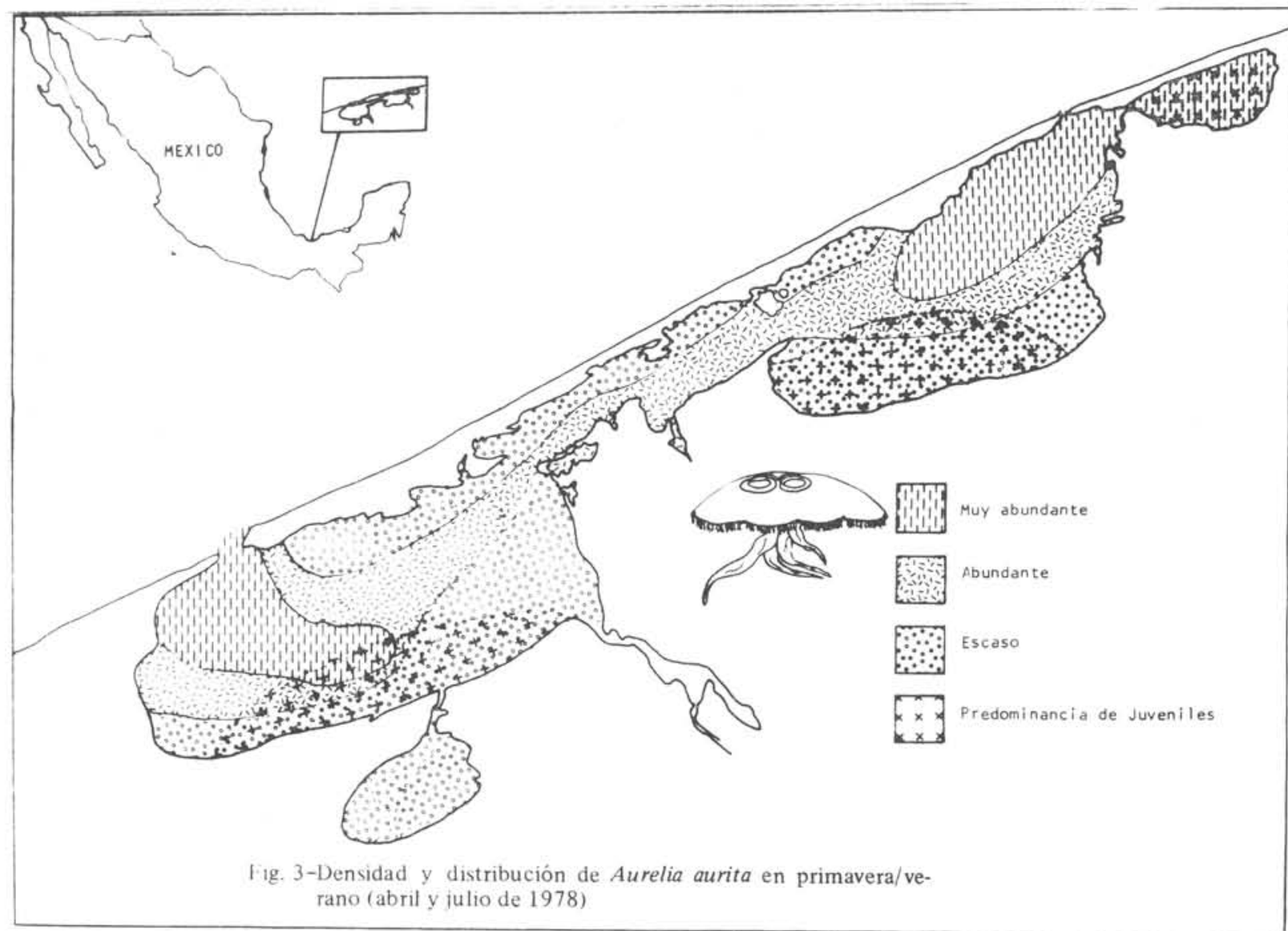

mentales: la relativa disminución de la temperatura por una parte, como fenómeno general del sistema, así como a la presencia de vientos del norte que actúan transportando a las medusas hacia los bajos y la costa en donde son depositadas en grandes cantidades, abatiéndose por lo tanto su alta densidad de población; por otra parte, también se puede atribuir a su salida del sistema debido a que esta es la época de défict en la interacción Laguna-Golfo y posiblemente estas poblaciones de $S$. meleagris busquen las condiciones de temperatura óptima. En este período se presentó una enorme densidad dada por las nuevas generaciones de medusas, siendo las más pequeñas de điciembre a febrero con tallas entre 10 y $25 \mathrm{~mm}$ de diámetro umbrelar, y de febrero a abril predominaron juveniles de 25 a $50 \mathrm{~mm}$. Entre los meses de abril a julio se observó el mayor crecimiento de estas medusas alcanzando las tallas de los adultos entre 100 y $200 \mathrm{~mm}$, con que se incrementó fuertemente la densidad de poblaciones de medusas grandes. No obstante, durante esta última época prevalecieron altas densidades de juveniles de 25 a $50 \mathrm{~mm}$, localizadas especialmente en las márgenes internas del sistema y en la Laguna Redonda.

\section{Aurelia aurita L.}

La presencia de formas adultas de esta especie sólo fue muy manifiesta en el período primavera/verano; en el resto del año las medusas grandes fueron muy escasas, sin embargo, los es. tadios juveniles sí se presentaron en todo el año, particularmente en las porciones sur de las Lagunas del Carmen, La Machona y en las Lagunas Redonda y La Palma. La cuantificación de A auri. ta se vió limitada debido a la transparencia de la propia médusa, a su tipo de movimiento, a su cualidad de agregación numerosa, así como por los reflejos del agua, lo que obligó a estimaciones muy relativas como-muy abundante, abundante, escaso y predominancia de juveniles, como se muestra en la Figura 3.

El patrón de distribución de $A$, aurita resulta altamente semejante al de $S$. meleagris, con incrementos cerca de las bocas en donde se observan los especímenes más grandes ( 200 a $400 \mathrm{~mm}$ ) y numerosas poblaciones de juveniles hacia las margenes interiores, con predominancia de tallas entre los 30 y $100 \mathrm{~mm}$ de diámetro umbrelar. aue en numerosas ocasiones saturaron las redes en arrastres de aproximadamente $100 \mathrm{~m}$ en la horizontal,

Durante el mes de abril de 1978 fueron observadas nuevas especies de medusas rizostómidas de diversa talla, destacando una especie con ejemplares de 400 a $600 \mathrm{~mm}$ de diámetro umbrelar y otras de tallas pequeñas entre 10 y $50 \mathrm{~mm}$, que trataremos posteriormente.

Estos resultados requieren de una amplia discusión, particularmente enfocada a confirmar o diferir de los de numerosos autores que han contribuido a la biología y ecología de las grandes medusas del Atántico Occidental y que en los espacios de esta contribución no es posible tratar por ahora.

\section{Reconocimientos}

Al compañero y fino amigo Andrés Reséndez Medina por su entusiasta cooperación.

\section{Referencías Bibliográficas}

GOMEZ-AGUIRRE, S. 1975. Observaciones comparativas de resultados de estudios del plancton de lagunas costeras del Golfo de México. Mem. II Simp. latinoam. Oceanogr. Biol. Universidad de Oriente, 24 al 28 de Noviembre de 1975, Cumaná, Venezuela, vol. 1, p.21-33, 4 figs.

-.-- Ingresos masivos de Stomolophus meleagris Agassiz (Scyphozoa-Rhizostomeae) en lagunas costeras de México. Mem.I Congr. nal Zool., 9 al 12 de Octubre de 1977. Escuela Nacional de Agricultura, Chapingo, México, (en prensa).

RESÉNDEZ-MEDINA, A. 1980. Hidrología de un sistema de lagunas costeras del Sur del Golfo de México en un período comprendido entre 1977/1978. Mem. V Simp. latinoam. Oceanogr. Biol. Instituto Oceanográfico, Universidade de São Paulo, 20 al 25 de Noviembro de 1978, São Paulo, Brasil. Bolm Inst. oceanogr., S Paulo, 29 (2): 